\title{
A digital nonlinear piezoelectric tuned vibration absorber
}

\author{
G Raze ${ }^{1}$, A Jadoul ${ }^{2}$, S Guichaux ${ }^{2}$, V Broun ${ }^{2}$ and G Kerschen ${ }^{1}$ \\ ${ }^{1}$ Space Structures and Systems Laboratory, Aerospace and Mechanical Engineering \\ Department, University of Liège, Quartier Polytech 1 (B52/3), Allée de la \\ Découverte 9, B-4000 Liège, Belgium \\ ${ }^{2}$ Service d'Electronique, Département Ingénieur Industriel, Haute Ecole de la \\ Province de Liège, Quai Gloesener 6, B-4020 Liège, Belgium \\ E-mail: g.raze@uliege.be
}

\begin{abstract}
This study presents the practical realization of a digital vibration absorber that, owing to the flexibility provided by the digital unit, synthesizes linear and nonlinear shunt circuits. The absorber, composed of a microprocessor and a current source, is connected to the host structure with piezoelectric patches. The performance of both circuits is compared experimentally for a nonlinear host structure. The superiority of a properly-tuned nonlinear absorber over its linear counterpart is validated, but the limits of the nonlinear absorber are also explored. Moreover, the accuracy of the tuning procedure and formulas is assessed through experimental parametric studies.
\end{abstract}

Keywords: piezoelectric vibration absorber, synthetic impedance, nonlinear vibrations, nonlinear vibration absorber, principle of similarity 


\section{Introduction}

Since the seminal works of Forward [1] and Hagood and von Flotow [2], piezoelectric shunt damping has become a widespread technique to reduce unwanted vibrations in structural systems. This approach relies on the transducing capability of a piezoelectric material, i.e., its ability to convert part of its mechanical energy into electrical energy. This electrical energy may then be dissipated by connecting properly tuned shunt circuits to the transducer. A popular shunt circuit is the resonant series RL one, in which the inductance and resistance are tuned so that the maximum vibratory amplitude of the controlled structure under harmonic loading is minimal. The exact inductance and resistance values leading to this $H_{\infty}$-optimal solution were derived by Soltani et al [3]. This strategy results in the so-called equal-peak design, because the frequency response function of the controlled structure exhibit two resonance peaks of equal amplitude. A recent paper by Ikegame et al [4] gathers tuning formulas for usual configurations and objectives at hand. Gripp and Rade [5] reviewed a number of works and applications of piezoelectric shunt damping. Interestingly, absorbers relying on the resonance of an added device also come in various forms and are used in a variety of domains. Examples include tuned mass dampers [6], Helmholtz resonators in acoustics [7] and plasmonic absorbers for opto-electronic devices $[8,9,10]$. Resonance can be a desirable feature in some applications, for instance high-performance infrared sensors [11].

Piezoelectric tuned vibration absorbers (PTVA) rely on a precise tuning of the electrical resonance frequency according to that of the host structure. The latter may be variable due to, e.g., time-varying characteristics or structural nonlinearities. Hence, this technique is often considered as lacking robustness. This is why Agnes and Inman [12] investigated the effect of adding nonlinear elements in the shunt circuit. They found that the bandwidth of the piezoelectric absorber could be increased; however, undesirable nonlinear phenomena such as quasiperiodic and chaotic motions could also be observed. Along the same lines, Richard et al utilized continuous switching of a piezoelectric shunt to realize a nonlinear absorber [13]. In [14], Zhou et al explored a piezoelectric nonlinear energy sink (NES) comprising an essential nonlinearity made of a ferroelectric capacitor and a negative capacitance circuit. Because this absorber has no preferential resonance frequency, it can pump the energy from the host structure in an irreversible fashion. This energy transfer is, however, conditioned upon an energy threshold, i.e., the transfer does not occur if the vibrational energy of the host structure is not large enough. Based on a nonlinear principle of similarity, Soltani and Kerschen [15] introduced a nonlinear PTVA. The absorber nonlinearity is similar to that present in the host structure, so that the host structure and absorber frequencies evolve in a similar way when the forcing amplitude changes, which, in turn, increases the range of forcing amplitudes over which the nonlinear PTVA is effective.

Focusing now on experimental demonstrations, a nonlinear absorber relying on the saturation phenomenon was realized through digital control in [16] and [17]. The first practical implementation of a nonlinear PTVA was achieved in [18] whereas Silva et 
al [19] realized a piezoelectric NES using analog electronics. An interesting feature of [18] is that the use of synthetic inductors (and, thus, of a power supply) was avoided, leading to a fully passive piezoelectric absorber. We also note that different nonlinear absorbers, including an NES and an NLTVA were realized experimentally using a tunable magnetic vibration absorber [20].

The objective of the present study is to realize PTVAs to mitigate structural vibrations through synthetic impedances, following the idea proposed by Fleming et al [21]. The innovation of this work is that the full flexibility of the digital absorber is exploited to implement arbitrarily complex nonlinear laws, something which is not easily achievable using purely mechanical elements, hence, paving the way for a more widespread use of nonlinear absorbers in real-world applications.

The paper is organized as follows. Section 2 briefly reviews the theory behind the tuning of linear and nonlinear PTVAs. Section 3 introduces the proposed digital absorber and discusses its various components. In Section 4, experimental tests of a digital linear absorber acting on a nonlinear host structure are reported. Section 5 then carries out the experimental demonstration of the digital nonlinear absorber for which different nonlinear (polynomial) laws are considered. Finally, the conclusions of the present study are drawn in Section 6.

\section{Piezoelectric tuned vibration absorbers}

\subsection{Linear piezoelectric tuned vibration absorber}

A resonant shunt circuit comprises an inductor and a resistor, arranged either in series or in parallel. Figure 1 depicts a single-degree-of-freedom (SDOF) mechanical oscillator with a piezoelectric stack connected to a series RL shunt circuit.

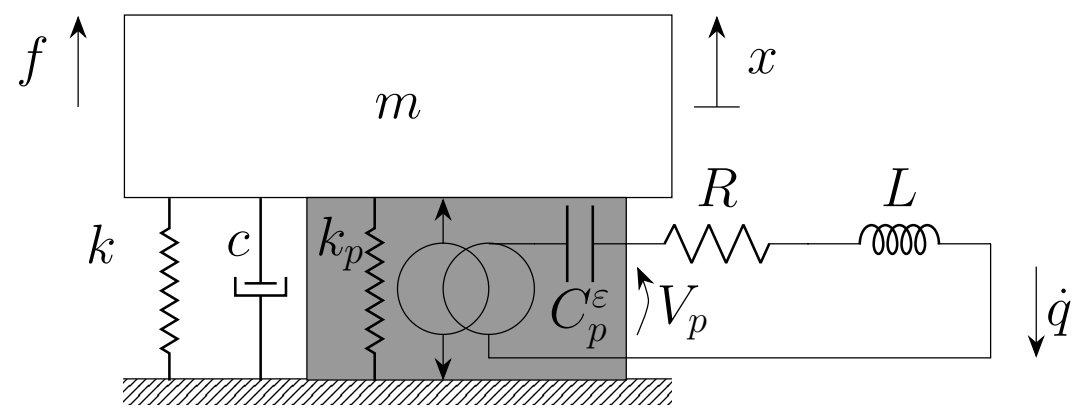

Figure 1: Mechanical oscillator with a piezoelectric transducer shunted by a series RL circuit.

The system is governed by the following equations [2]

$$
\left\{\begin{array}{c}
m \ddot{x}+c \dot{x}+k_{o c} x-\theta q=f \\
L \ddot{q}+R \dot{q}=V_{p}
\end{array},\right.
$$


where the voltage across the piezoelectric transducer $V_{p}$ is given by

$$
V_{p}=\theta x-\frac{q}{C_{p}^{\varepsilon}},
$$

Parameters $m$ and $c$ are the mass and damping of the SDOF oscillator, respectively, $k_{o c}=k+k_{p}$ is the total structural stiffness (with $k$ the stiffness of the structure without piezoelectric transducer, and $k_{p}$ the stiffness of the piezoelectric transducer when it is open-circuited), $x$ is the displacement of the mechanical oscillator, $\theta$ is a coupling coefficient, $f$ is the external forcing, $L$ and $R$ are the inductance and resistance of the shunt circuit, respectively, $C_{p}^{\varepsilon}$ is the piezoelectric capacitance at constant strain and $q$ is the electrical charge flowing through the piezoelectric transducer. An upper dot denotes time derivation.

The shunt circuit parameters can be tuned in a number of ways according to the objectives at hand, see, e.g., the recent paper by Ikegame et al [4]. Because this study considers harmonic forcing, the $H_{\infty}$-optimum tuning given by Soltani et al [3] is adopted. The generalized electromechanical coupling factor $K_{c}$ is given by [2]

$$
K_{c}^{2}=\frac{\omega_{o c}^{2}-\omega_{s c}^{2}}{\omega_{s c}^{2}}
$$

where $\omega_{s c}$ and $\omega_{o c}$ are the angular resonance frequencies of the structure when the piezoelectric transducer is short-circuited and open-circuited, respectively. An intermediate variable $r$ is introduced as

$$
r=\frac{\sqrt{64-16 K_{c}^{2}-26 K_{c}^{4}}-K_{c}^{2}}{8}
$$

which is related to the equal-peak amplitude $h$ through $h=\left(1+K_{c}^{2}\right) /\left(k_{o c} \sqrt{1-r^{2}}\right)$. The optimal inductance and resistance values are then given by

$$
L=\frac{4 K_{c}^{2}+4}{3 K_{c}^{2}-4 r+8} \frac{1}{\omega_{o c}^{2} C_{p}^{\varepsilon}}
$$

and

$$
R=\frac{2 \sqrt{2\left(K_{c}^{2}+1\right)\left(27 K_{c}^{4}+K_{c}^{2}(80-48 r)-64(r-1)\right)}}{\left(5 K_{c}^{2}+8\right) \sqrt{3 K_{c}^{2}-4 r+8}} \frac{1}{\omega_{o c} C_{p}^{\varepsilon}},
$$

respectively $[3,4]$. For illustration, considering the linear parameters of the experimental set-up described in Section 4 and listed in Table 1, the PTVA parameters in Table 2 are obtained. Eventually, this tuning methodology yields the so-called equal-peak design, as illustrated in Figure 2.

\subsection{Nonlinear piezoelectric vibration absorber}

When the host structure is nonlinear, a linear PTVA can quickly become detuned due to its narrow bandwidth. The purposeful introduction of nonlinearity in the PTVA and the subsequent harnessing of its behavior allows the absorber to remain tuned to the changing frequency of the host structure [15]. For this purpose, the functional form of 


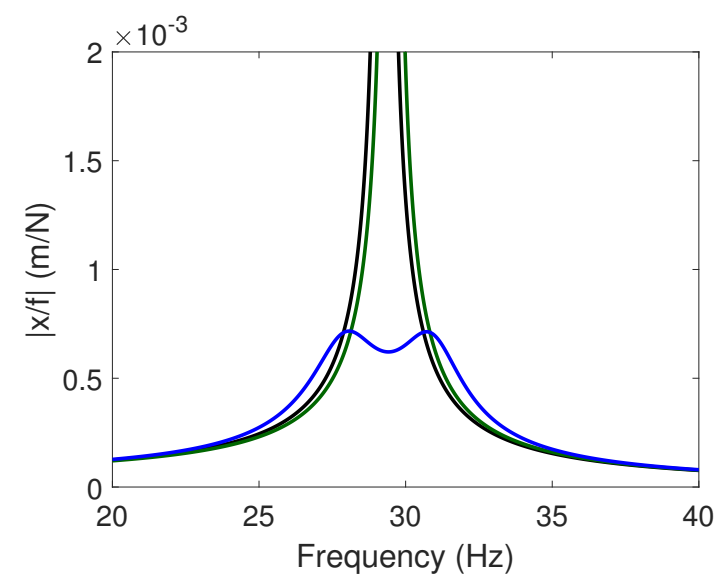

Figure 2: Frequency response function of the system in Figure 1: short-circuited piezoelectric transducer $(-)$, open-circuited piezoelectric transducer $(-)$ and equalpeak design $(-)$.

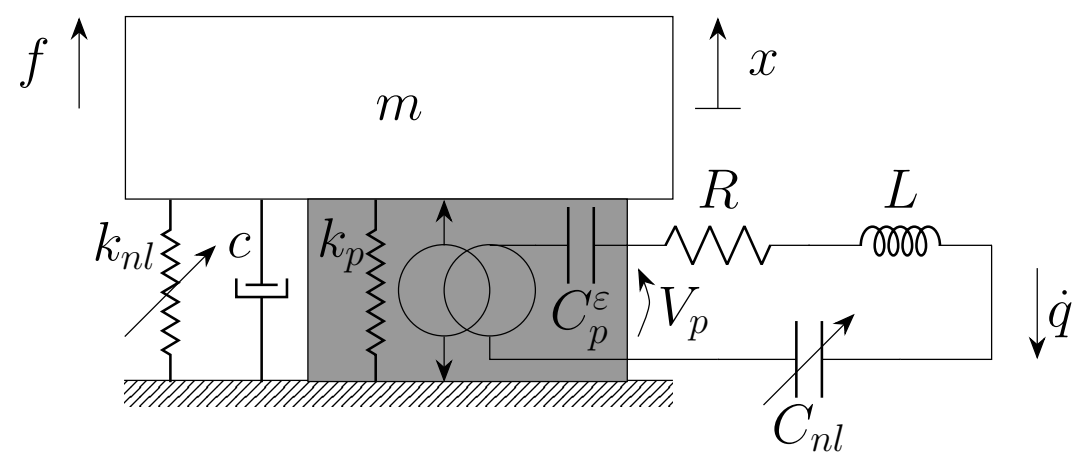

Figure 3: Nonlinear oscillator with a piezoelectric transducer shunted by a series RL circuit possessing a nonlinear capacitor.

the nonlinearity in the PTVA is to be chosen according to a principle of similarity [22], i.e., it should be identical to that of the host structure.

For a nonlinear shunt circuit coupled to a Duffing oscillator, depicted in Figure 3, the governing equations read

$$
\left\{\begin{aligned}
m \ddot{x}+c \dot{x}+k_{o c} x+k_{3} x^{3}-\theta q & =f \\
L \ddot{q}+R \dot{q}+C_{3} q^{3} & =V_{p}
\end{aligned}\right.
$$

where $k_{3}$ is the cubic stiffness coefficient and $C_{3}$ is the cubic capacitance coefficient $\left(C_{3}=0\right.$ for a linear shunt circuit) [12]. This latter coefficient can be determined from the parameters of the system using the simplified formula in [18]:

$$
C_{3}=\frac{2 L^{2}}{m^{2}} k_{3}
$$

Figure 4 shows the nonlinear frequency responses (NFRs) of the Duffing oscillator controlled by either a linear (Figure 4a) or nonlinear (Figure 4b) PTVA. These responses were computed using a harmonic balance formalism coupled with a continuation 
procedure [23]. As the forcing amplitude increases, the linear absorber becomes severely detuned, which generates detrimental vibrations. Conversely, the nonlinear PTVA performance remains unchanged for the different forcing amplitudes.

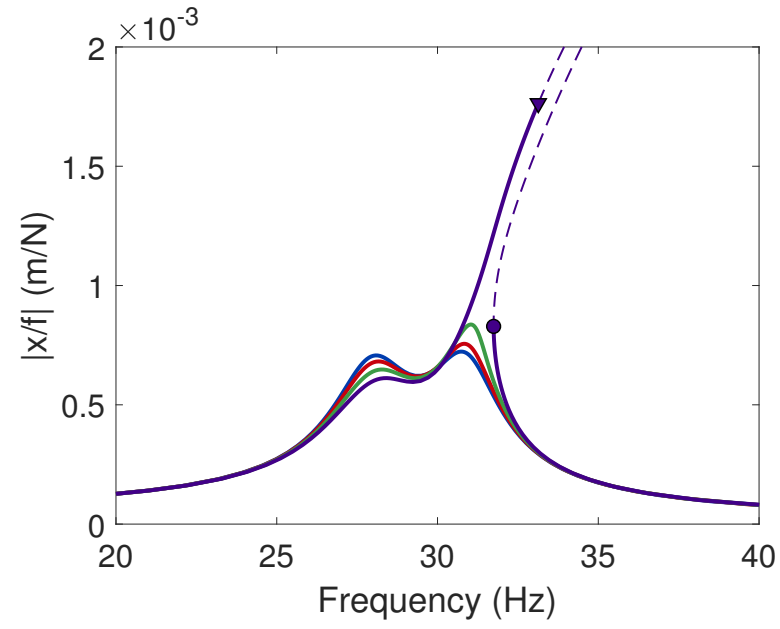

(a)

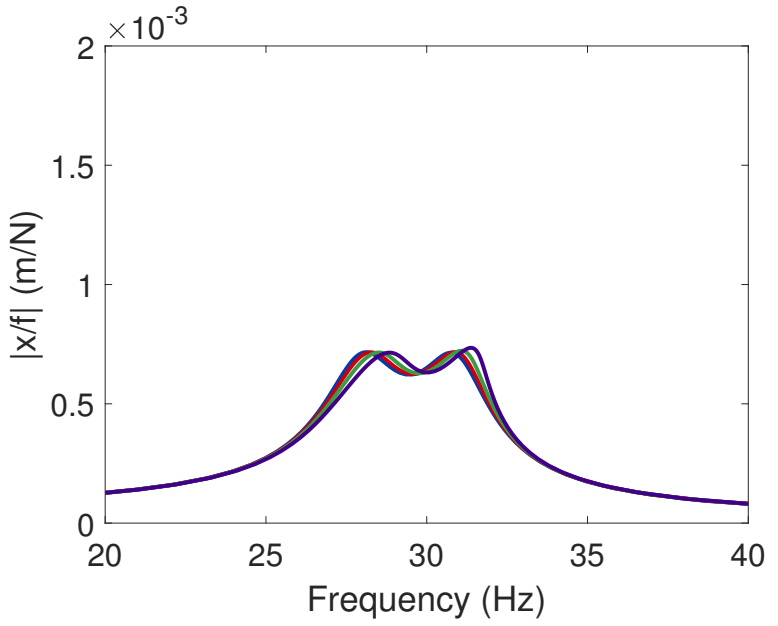

(b)

Figure 4: NFRs of a Duffing oscillator controlled by a linear (a) and nonlinear (b) PTVA: $0.2 \mathrm{~N}(-), 0.4 \mathrm{~N}(-), 0.6 \mathrm{~N}(-), 0.8 \mathrm{~N}(-)$. - : stable solution, - - : unstable solution, $\bullet$ : fold bifurcation, $\mathbf{\nabla}$ : Neimark-Sacker bifurcation.

\section{A digital piezoelectric vibration absorber}

When it comes to the practical realization of a PTVA, a number of challenges have to be overcome. The first difficulty is the large inductance value (typically hundreds of Henries) required for structures with low resonance frequencies. Although physical inductors with such high inductance values were realized [24], they are not commercially available. A common workaround is to use synthetic inductors or gyrators, but they come with non-ideal characteristics, such as frequency-dependent resistance [25]. Another challenge pertaining to nonlinear absorbers is the tailoring of the nonlinear behavior of the electrical components so as to obey the principle of similarity. Even if this problem was addressed for cubic nonlinearities through the use of saturable inductors [18], there is not much variety in the functional forms that can be realized with electrical components.

The concept of synthetic impedance proposed by Fleming et al [21] solves these issues altogether. A digital signal processing unit together with a current source make the realization of an arbitrary impedance possible. This however comes at the expense of a non-fully-passive absorber (because the analog and digital electronics need power) and at the risk of creating instabilities (because the system contains active elements). The realization of a synthetic impedance mimicking a PTVA is discussed in what follows. 


\subsection{Hardware}

The digital vibration absorber used in this study comprises two parts, namely a digital processing unit and an analog board. The digital processing unit implements the desired input-output $(\mathrm{I} / \mathrm{O})$ relation between the voltage across the electrodes of the piezoelectric transducer and its current. The analog board serves as the interface between the piezoelectric transducer and the digital processing unit.

The schematic of the digital absorber is depicted in Figure 5. It is very similar to that used by Matten et al [26]. The patch voltage $V_{p}$ is measured with a voltage divider made of $R_{1}$ and $R_{2}$. The operational amplifier OA1 is a follower [27] which outputs a fraction of the patch voltage $\alpha V_{p}$, where $\alpha=R_{2} /\left(R_{1}+R_{2}\right)$. A constant offset voltage $\Delta V_{\text {in }}$ is generated by a shunt voltage regulator to comply with the input voltage range of the analog-to-digital converter (ADC) of the microcontroller unit (MCU). OA2 sums the two voltages and outputs $\alpha V_{p}+\Delta V_{i n}$. The MCU computes the input-output relation to inject the desired current and sends a voltage signal with its digital-to-analog converter (DAC) to OA3, whose output simply follows the input. Since the output voltage range of the MCU may not be symmetric with respect to the circuit ground, another offset voltage $\Delta V_{\text {out }}$ is generated by a shunt voltage regulator. OA4 subtracts this offset to the output of OA3. This results in an output voltage signal $V_{\text {out }}$ proportional to the current to be injected in the transducer. This voltage signal is imposed on one pole of the resistor $R_{i}$, while the other one is maintained to a virtual ground by OA5. Thus, the current injected into the piezoelectric patch is $V_{\text {out }} / R_{i}$.

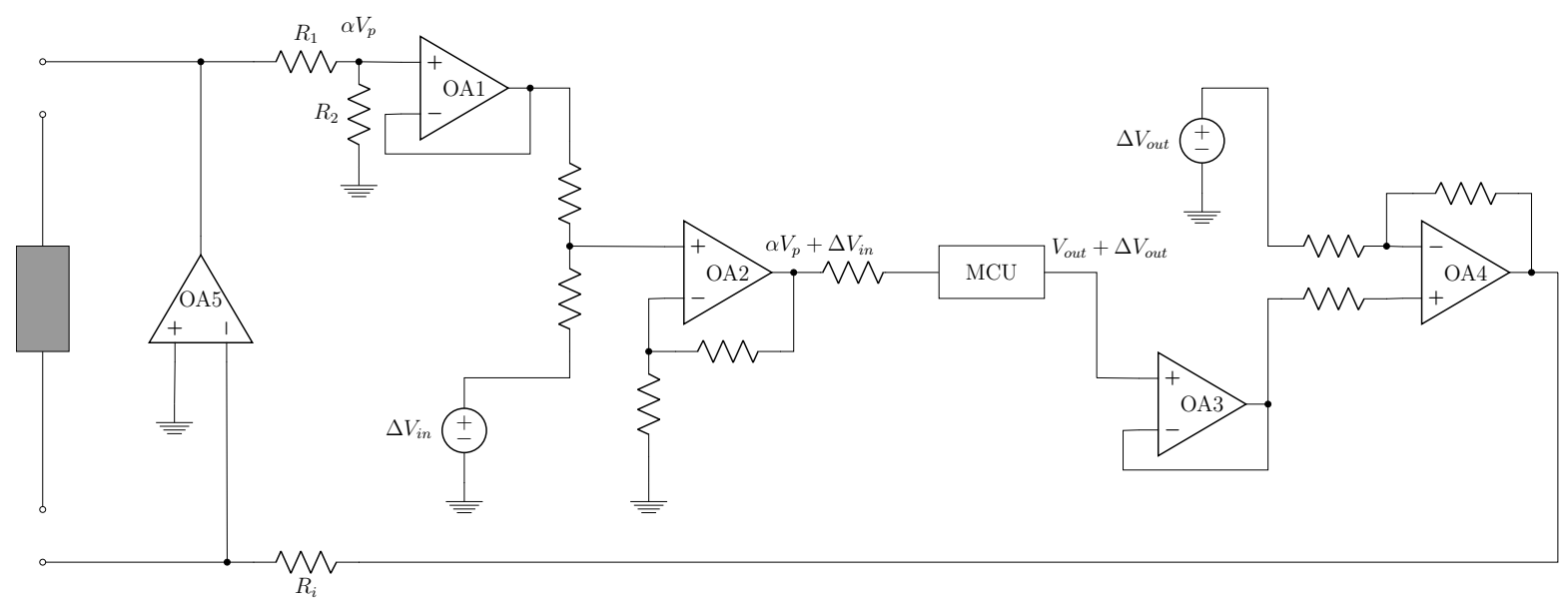

Figure 5: Layout of the digital absorber. The gray box represents the piezoelectric transducer.

The MCU used in this work is an Arduino Due. This microcontroller was chosen for its price, availability, ease to use and the presence of DACs. The operational amplifier OA5, a PA78 power operational amplifier from APEX Microtechnology [28], is able to cope with the high voltages generated by the piezoelectric transducer, which went up to $\pm 150 \mathrm{~V}$. 


\subsection{Software}

The Arduino Due can easily be programmed to implement an arbitrary I/O relation with MATLAB Simulink. The I/O relation may be represented as a block diagram, and the support packages for Arduino Hardware were used to generate the code to be uploaded to the microcontroller in a fully automated way.

3.2.1. Linear PTVA Implementing a linear PTVA is fairly straightforward, as depicted in Figure 6. The ADC reads a voltage level and translates it into an unsigned integer number. This integer is reconverted to a floating point number representing a voltage level by the gain $g_{i n}$. An offset $\Delta V_{i n}$ is added to compensate for the input voltage offset generated by the analog board. The shifted and scaled signal is processed according to the desired I/O relation $Y$, the synthesized shunt circuit admittance [21]. It is then amplified by a gain $g$ whose purpose is explained hereafter. The output signal is passed through a saturation operator to avoid integer overflow at the DAC. Finally, the output signal is shifted (by $\Delta V_{\text {out }}$ ) and scaled (by $g_{\text {out }}$ ) back to an unsigned integer to be fed to the DAC.

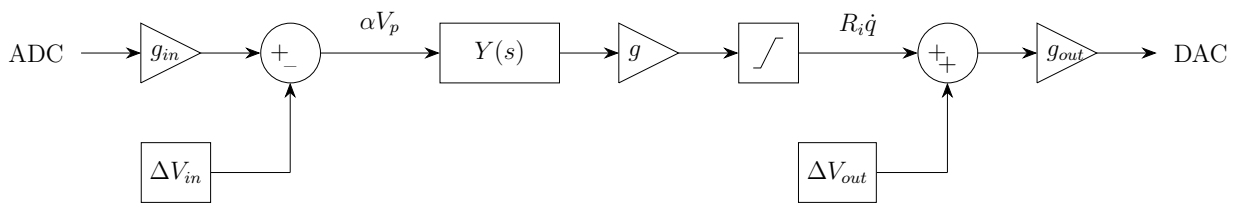

Figure 6: Block diagram of the synthesized linear PTVA.

If properly tuned, every voltage offset from the analog board should be compensated by an offset in the software. The remaining parameter to tune is the gain $g$ whose purpose is to ensure the consistency of the I/O relation with a shunt circuit from the point of view of the transducer. If the offsets are perfectly compensated, the current $i=\dot{q}$ injected into the transducer should be

$$
i(s)=\frac{1}{R_{i}} V_{\text {out }}(s)=\frac{g \alpha}{R_{i}} Y(s) V_{p}(s),
$$

where $s$ is the Laplace variable. Equation (9) shows that if the emulated shunt circuit is to mimic the desired admittance, the following consistency condition should be ensured

$$
\frac{g \alpha}{R_{i}}=1
$$

3.2.2. Nonlinear PTVA The nonlinear voltage can be computed if the charge flowing through the piezoelectric transducer is known. It can be retrieved from the output voltage since

$$
V_{\text {out }}=R_{i} \dot{q}
$$

Moreover, using Kirchhoff's voltage law,

$$
\alpha V_{p}=\alpha V_{L}+\alpha V_{N L},
$$


where $V_{L}=L \ddot{q}+R \dot{q}$ is the voltage across the RL circuit and $V_{N L}=C_{3} q^{3}$ is the voltage across the nonlinear capacitor. Then, Equation (11) and Equation (12) suggest that the nonlinear shunt circuit may be implemented by adding a nonlinear feedback [29] to the block diagram in Figure 6, as depicted in Figure 7.

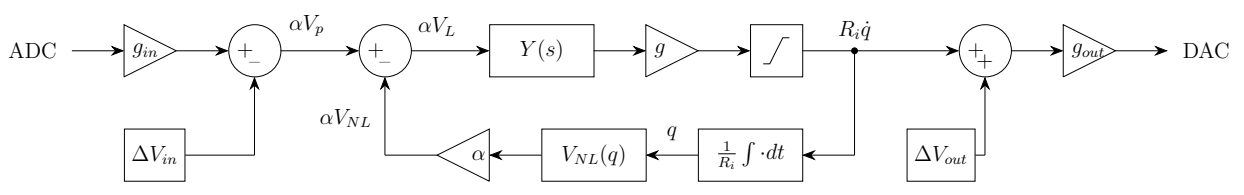

Figure 7: Block diagram of the synthesized nonlinear PTVA.

Small signal offsets are unavoidable in practice. This causes a drift of the integrator and an eventual saturation of the signals. As a remedy, a lossy integrator (i.e., a firstorder lowpass filter) or a bandpass filter can replace the integrator. The passband of such filters should be in a low frequency range, such that the filter behaves as an integrator in the frequency range of interest. The advantage of using a bandpass filter over a lossy integrator is its ability to completely reject DC offsets, preventing asymmetric AC signal distortion.

\section{Experimental demonstration of a digital linear PTVA}

\subsection{Experimental setup}

The structure under investigation is a clamped-free beam with a clamped thin lamina attached to its free end, as shown in Figure 8. Due to its large relative deformation, the thin lamina causes an overall hardening nonlinear structural behavior, which can accurately be modeled using a cubic spring. The structure is excited near its free end by an electrodynamic shaker (TIRA TV 51075). An impedance head (DYTRAN 5860B) is used to measure the force applied to the structure and the corresponding acceleration. The measurements are recorded by an acquisition system (LMS Scadas Mobile).

The beam is covered over its whole length with ten cells, composed of pairs of stacks of two PSI-5A4E piezoelectric patches, each pair being placed on either side of the beam. The five cells closest to the clamped end are connected in parallel and used for mitigating the resonant vibrations around the first beam mode, whereas the five other cells are left in open circuit. More details about the experimental setup can be found in [18].

The digital absorber connected to the patches is powered by a high-voltage (HV) power supply with $\pm 150 \mathrm{~V}$ (to power the operational amplifier OA5 in Figure 5) and with a low-voltage (LV) power supply with $\pm 10 \mathrm{~V}$ (to power the interface with the microcontroller unit). The microcontroller is programmed and powered via USB.

The voltage division ratio $\alpha=0.0109$ was set so that a maximum input voltage at the $\mathrm{MCU}( \pm 1.65 \mathrm{~V})$ corresponds nearly to a maximum output voltage of OA5 $( \pm 150 \mathrm{~V})$. The current injector resistance $R_{i}=152.9 \Omega$ was measured with a multimeter (FLUKE 


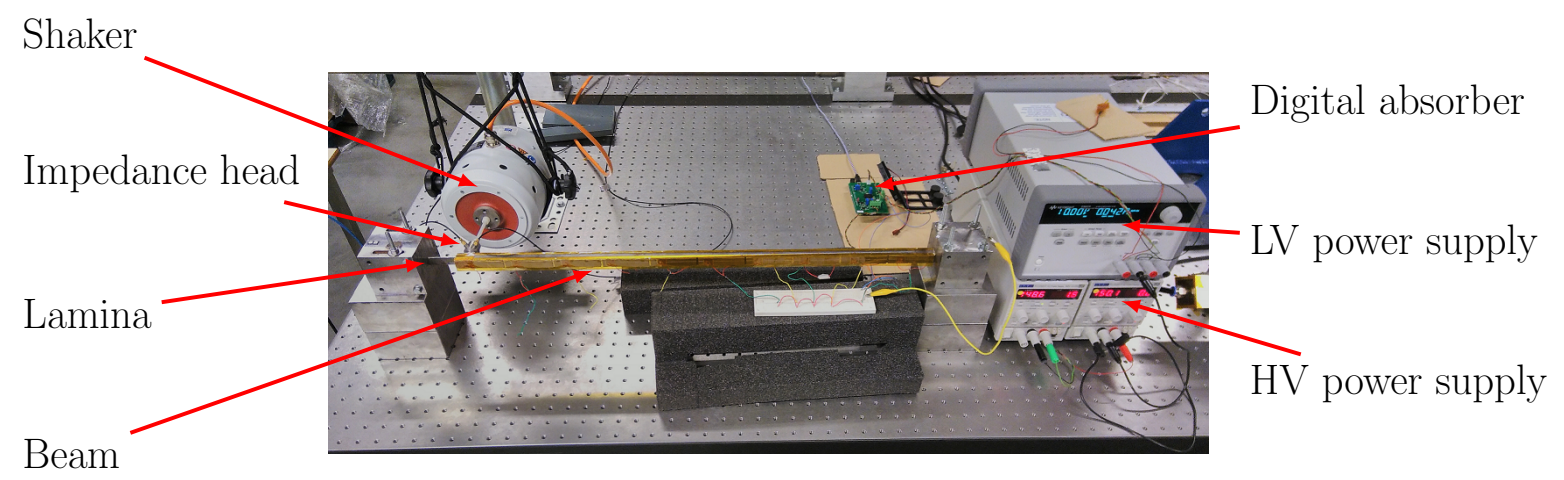

Figure 8: Picture of the experimental setup.

177). The gain $g=14,021$ was deduced from Equation (10). The voltage offsets $\Delta V_{\text {in }}$ and $\Delta V_{\text {out }}$ were both set to $1.65 \mathrm{~V}$, so that the full $\mathrm{ADC}$ and $\mathrm{DAC}$ ranges of the $\mathrm{MCU}$ could be used.

\subsection{System identification}

The first step to tune the shunt circuit is to identify the parameters of the host system. The piezoelectric capacitance was measured with a multimeter (FLUKE 177). The linear mechanical parameters were determined from a low-level broadband excitation. A first test was performed with open-circuited patches $(q=0)$. The obtained frequency response function (FRF) was fitted to obtain a minimum least-squares error between the model and the measurements, giving an estimate of the parameters $m, c$ and $k_{o c}$. The process was repeated with short-circuited patches, giving an estimation of $k_{s c}$, the structural stiffness when the piezoelectric patches are short-circuited $\left(V_{p}=0\right)$. From Equation (1) and Equation (2), it can be derived that the following relation holds:

$$
k_{s c}=k_{o c}-\theta^{2} C_{p}^{\varepsilon}
$$

so that $\theta$ can be deduced from the estimated quantities. The system has a generalized electromechanical coupling factor $K_{c}=12.43 \%$. Table 1 contains the identified parameters. Figure 9 shows an excellent agreement between the experimental and predicted FRFs.

The nonlinear stiffness of the thin lamina was estimated by exciting the system with short-circuited patches at higher forcing amplitudes. The excitation was a stepped sine with a controlled amplitude of $0.2 \mathrm{~N}$ between 28 and $32 \mathrm{~Hz}$. Following [18], the recorded force and acceleration signals were digitally bandpass filtered and gathered in vectors f and $\ddot{\mathbf{x}}$, respectively. The velocity and displacement vectors were estimated by time integration. Rewriting the first line of Equation (7)

$$
\mathbf{x}^{3} k_{3}=\mathbf{f}-[\ddot{\mathbf{x}}, \dot{\mathbf{x}}, \mathbf{x}]\left[\begin{array}{c}
m \\
c \\
k_{s c}
\end{array}\right]
$$




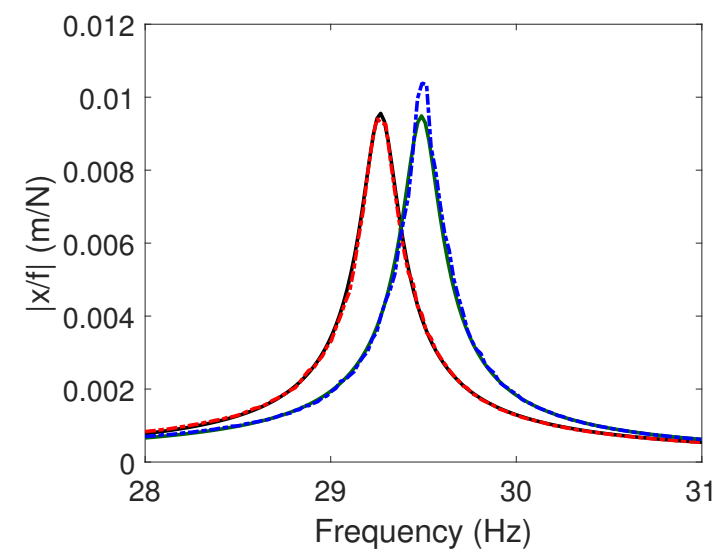

Figure 9: Experimental and predicted FRFs: model (sc: - oc: - ) and measurements (sc: ---, oc: ---).

Table 1: Identified parameters of the experimental setup.

\begin{tabular}{llllllll}
\hline Characteristic & $m(\mathrm{~kg})$ & $c(\mathrm{Ns} / \mathrm{m})$ & $k_{s c}(\mathrm{~N} / \mathrm{m})$ & $k_{o c}(\mathrm{~N} / \mathrm{m})$ & $k_{3}\left(\mathrm{~N} / \mathrm{m}^{3}\right)$ & $C_{p}^{\varepsilon}(\mathrm{nF})$ & $\theta(\mathrm{N} / \mathrm{C})$ \\
\hline Value & 0.4464 & 0.5690 & $1.4975 \times 10^{4}$ & $1.5207 \times 10^{4}$ & $2.5046 \times 10^{9}$ & 250 & $3.04248 \times 10^{4}$ \\
\hline
\end{tabular}

shows that the nonlinear force vector $\mathbf{x}^{3} k_{3}$ can be calculated from the knowledge of the linear parameters at each time instant. It is represented by the black dots in Figure 10. Eventually, the nonlinear coefficient $k_{3}$ in Table 1 was identified through simple leastsquares fitting of the nonlinear force with a cubic law.

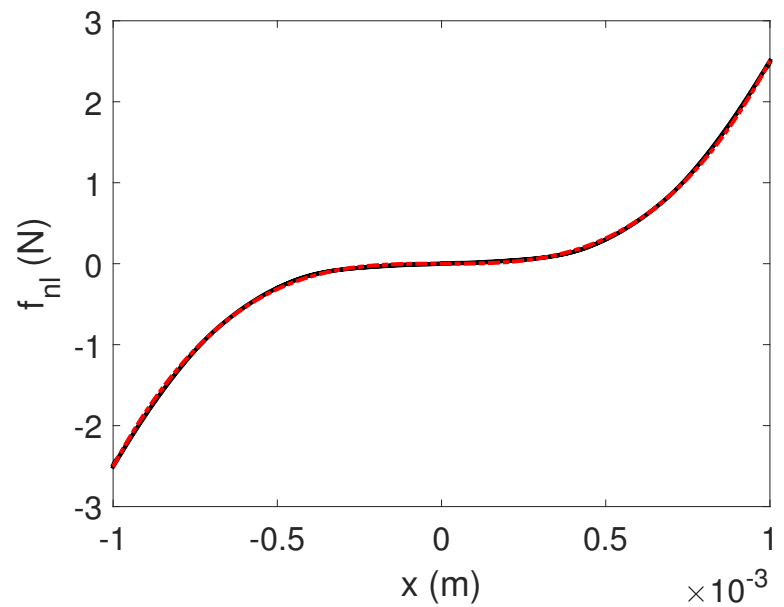

Figure 10: Nonlinear stiffness curve: measurement (•) and fitted cubic law (-•-).

\subsection{Synthesized admittance}

Based on the previous system identification, the parameters of the digital linear PTVA in Table 2 were computed using Equation (5) and Equation (6). The slight variability 
Table 2: Parameters of the linear and nonlinear PTVAs.

\begin{tabular}{llll}
\hline Parameter & $L(\mathrm{H})$ & $R(\Omega)$ & $C_{3}\left(V / C^{3}\right)$ \\
\hline Value & 117.42 & 3295 & $3.4022 \times 10^{14}$ \\
\hline
\end{tabular}

of the results from one test to another led to adaptations of the inductance $L$ of the order of $1 \%$ of the theoretical value. This adaptation was carried out to obtain equal peaks at the lowest forcing amplitude, namely $f=0.2 \mathrm{~N}$. The shunt circuit admittance was synthesized in the MCU after transforming the continuous admittance

$$
Y(s)=\frac{1}{L s+R}
$$

into a discrete transfer function using Tustin's method. The sampling frequency of the MCU was $10 \mathrm{kHz}$.

The input and output signals were measured by the MCU and recorded by a computer. The piezoelectric charge and current time derivative were estimated offline by time integration and time derivation of the output signal, respectively. Figure 11 and Figure 12, which compare the theoretical and post-processed voltages across the resistor and inductor, respectively, confirm that the MCU implements the desired input-output transfer function.

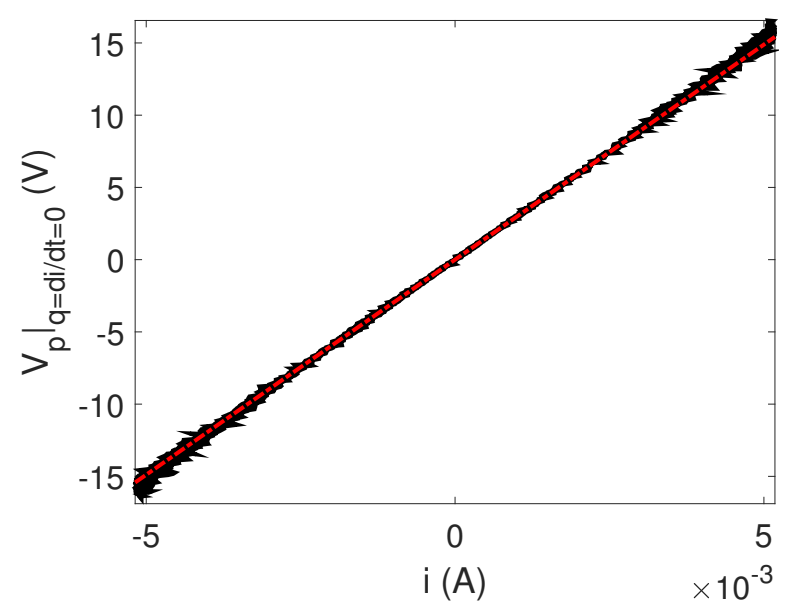

Figure 11: Voltage across the resistor: theoretical law (---) and measurements $(\bullet)$.

\subsection{Performance of the linear PTVA}

The experimental NFRs for forcing levels ranging from $0.2 \mathrm{~N}$ to $0.8 \mathrm{~N}$ are presented in Figure 13a. At $0.2 \mathrm{~N}$, the nonlinearity of the thin lamina is not activated; the linear PTVA gives rise to two equal peaks in the response. As the forcing level is increased, a clear detuning of the linear PTVA is observed. The comparison with the theoretical NFRs in Figure 4a reveals that the detuning is in fact more important than predicted. 


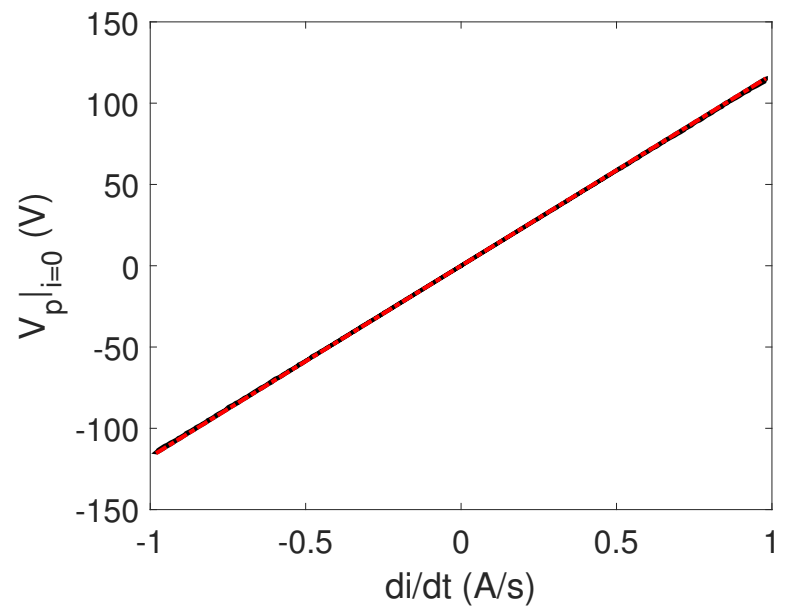

Figure 12: Voltage across the inductor: theoretical law (---) and measurements $(\bullet)$.

After detailed investigations, this detuning was attributed to the nonlinear behavior of the piezoelectric patches, as in [18]. The model in Equation (2) was thus upgraded using a negative cubic capacitance $C_{p, 3}$ and a positive cubic resistance $R_{p, 3}$, so that

$$
V_{p}=\theta x-\frac{1}{C_{p}^{\varepsilon}} q-C_{p, 3} q^{3}-R_{p, 3} \dot{q}^{3},
$$

with values given in Table 3 . The NFRs predicted through this updated model in Figure $13 \mathrm{~b}$ are now in close agreement with the experimental NFRs in Figure 13a.

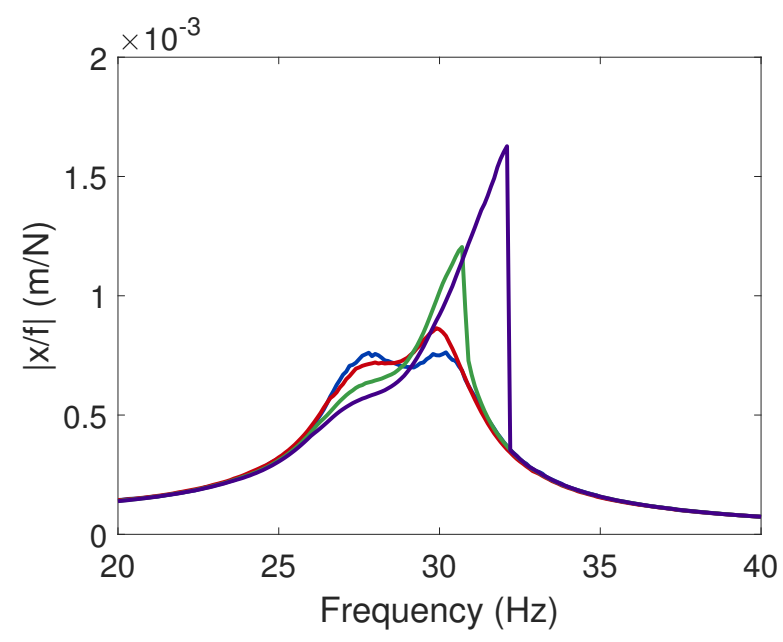

(a)

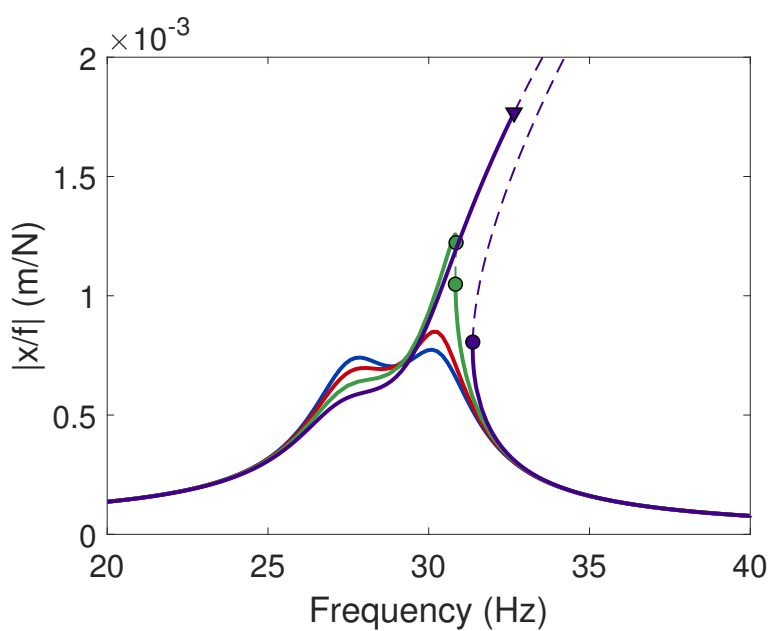

(b)

Figure 13: Experimental (a) and theoretical (updated) NFRs (b) of the nonlinear beam with a digital linear PTVA: $0.2 \mathrm{~N}(-), 0.4 \mathrm{~N}(-), 0.6 \mathrm{~N}(-), 0.8 \mathrm{~N}(-)$ - - : stable solution, - - : unstable solution, $\bullet$ : fold bifurcation, $\mathbf{\nabla}:$ Neimark-Sacker bifurcation. 
Table 3: Identified nonlinear parameters of the piezoelectric transducer.

\begin{tabular}{lll}
\hline Characteristic & $C_{p, 3}\left(\mathrm{~V} / \mathrm{C}^{3}\right)$ & $R_{p, 3}\left(\mathrm{~V} / \mathrm{A}^{3}\right)$ \\
\hline Value & $-2.3629 \times 10^{14}$ & $1.5352 \times 10^{7}$ \\
\hline
\end{tabular}

\section{Experimental demonstration of a digital nonlinear PTVA}

\subsection{Synthesized admittance}

To address the detuning of the linear PTVA in nonlinear regimes of motion, a cubic capacitance was introduced in the synthetic admittance $\left(\alpha V_{N L}\right.$ in Figure 7$)$. Accounting for the nonlinear behavior of the piezoelectric transducer, the value of the nonlinear capacitance was calculated to be $C_{3}-C_{p, 3} \approx 1.7 C_{3}$. The measured voltage of the nonlinear capacitor in Figure 14 was found to closely follow the law prescribed by the design.

We note that, to fully comply with the theoretical work of [15], a negative cubic resistance should also have been added to the synthesized shunt circuit to compensate for the positive cubic resistance in Equation (16), but this was avoided to prevent any risk of instability caused by such an active component. The linear resistance was nevertheless diminished by $10 \%$ in an attempt to limit the dissipation in the circuit at high forcing amplitudes. As shall be shown in Subsection 5.4, this has a limited impact on the performance of the digital nonlinear PTVA at low forcing levels.

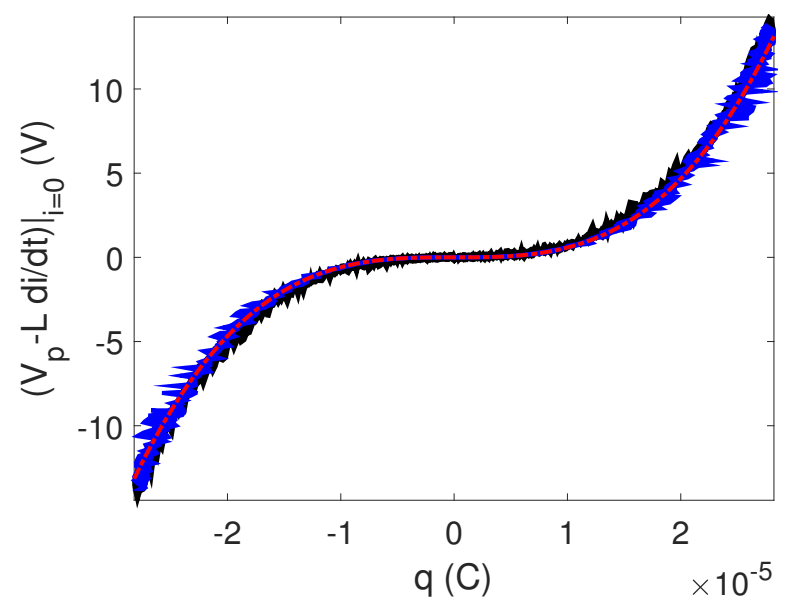

Figure 14: Voltage across the nonlinear capacitor: theoretical law (---), direct measurements of $V_{n l}(\bullet)$ and post-processed measurements from the input and output signals $(\bullet)$. 


\subsection{Performance of the nonlinear PTVA}

Figure 15a displays the experimental NFRs for forcing levels ranging from $0.2 \mathrm{~N}$ to $0.8 \mathrm{~N}$. Clearly, the nonlinear PTVA is able to maintain equal peaks in the frequency response for all forcing levels considered, confirming its superior performance over its linear counterpart. Furthermore, these experimental curves are in excellent agreement with the theoretical NFRs in Figure 15b.

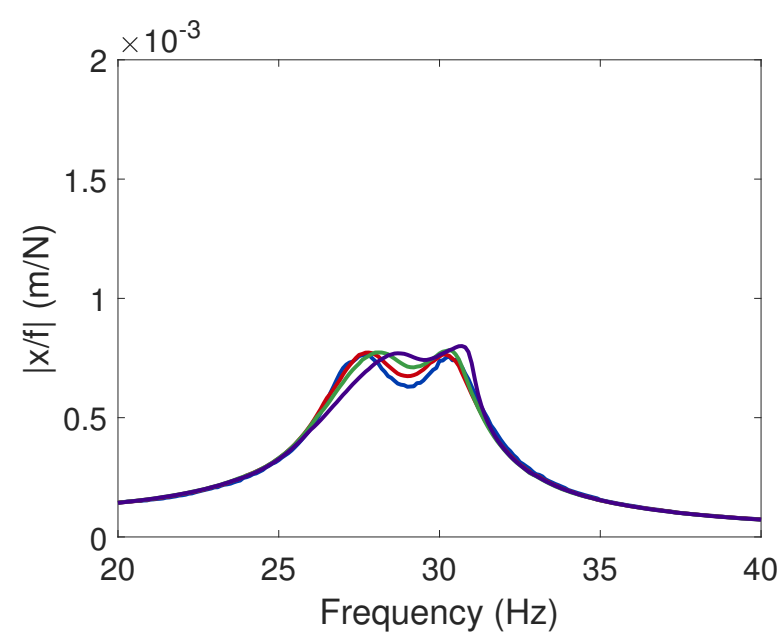

(a)

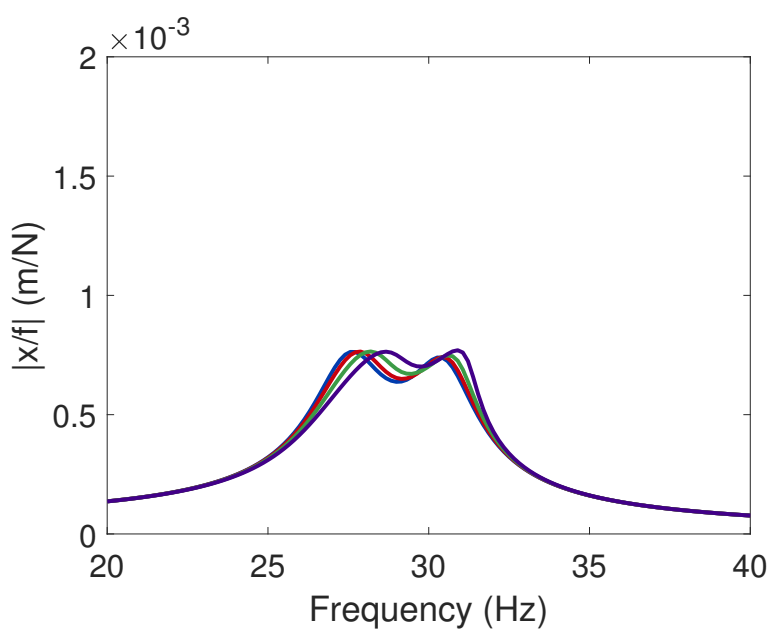

(b)

Figure 15: Experimental (a) and theoretical NFRs (b) of the nonlinear beam with a digital nonlinear PTVA: $0.2 \mathrm{~N}(-), 0.4 \mathrm{~N}(-), 0.6 \mathrm{~N}(-), 0.8 \mathrm{~N}(-)$.

A series of tests at higher forcing amplitudes was performed to investigate the potentially adverse dynamics brought by the nonlinear PTVA. Because of the detuning of the linear PTVA, these tests were not performed with this latter absorber in order to avoid the rupture of the thin lamina. Figure 16a depicts that the nonlinear PTVA starts to be slightly detuned at $1 \mathrm{~N}$, with a more pronounced detuning at $1.2 \mathrm{~N}$. The theoretical response in Figure 16b indicates that a detached resonance curve merges with the main response, marking the end of the working range of the nonlinear PTVA [15].

The digital nonlinear PTVA suffers from another limitation depicted in Figure 17. At $1.2 \mathrm{~N}$, the input signal of the MCU reaches its saturation limits, meaning that the operational amplifier OA5 in Figure 5 saturates correspondingly. The output signal saturates as well. This issue can be resolved by decreasing $R_{i}$, thereby decreasing $g$ by virtue of Equation (10), and thus the magnitude of the output signal, but this was not attempted in this work since the input voltage saturates anyway.

\subsection{Validation of the principle of similarity}

Quadratic, $C_{2} \operatorname{sign}(q) q^{2}$, and quintic, $C_{5} q^{5}$, nonlinear capacitances were also considered separately in the MCU. After fitting the cubic nonlinear restoring force of the thin lamina with quadratic and quintic laws, as shown in Figure 18, the coefficient of the 


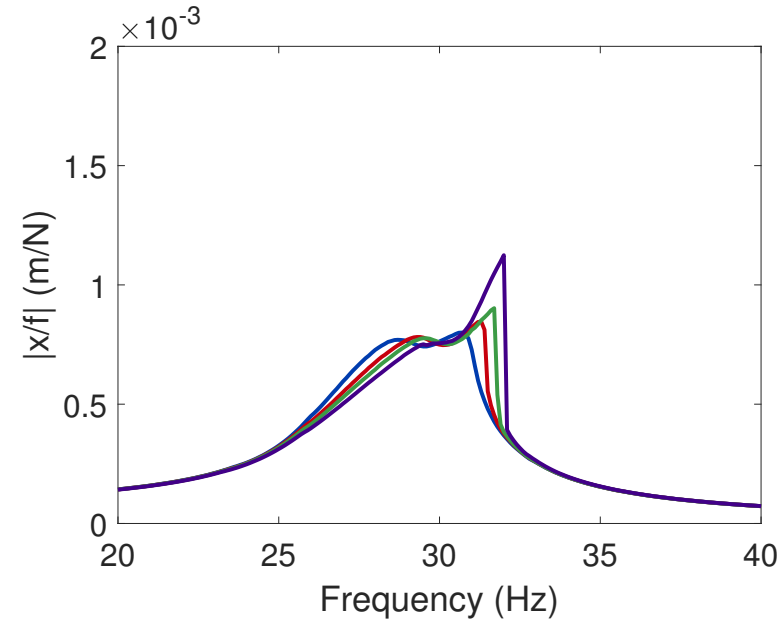

(a)

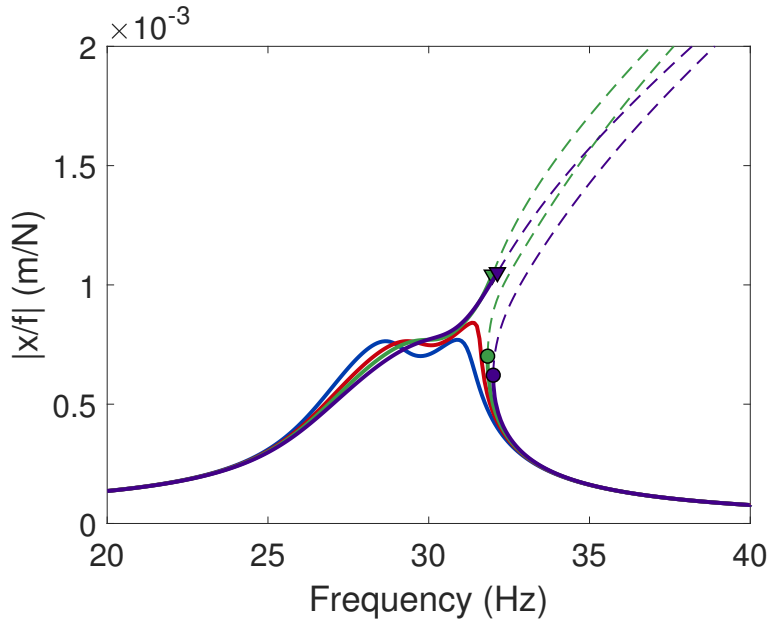

(b)

Figure 16: Experimental NFRs (a) and theoretical NFRs (b) of the nonlinear beam with a digital nonlinear PTVA: $0.8 \mathrm{~N}(-), 1.0 \mathrm{~N}(-), 1.1 \mathrm{~N}(-), 1.2 \mathrm{~N}(-)$. - : stable solution, - - : unstable solution, $\bullet$ : fold bifurcation, $\mathbf{\nabla}:$ Neimark-Sacker bifurcation.

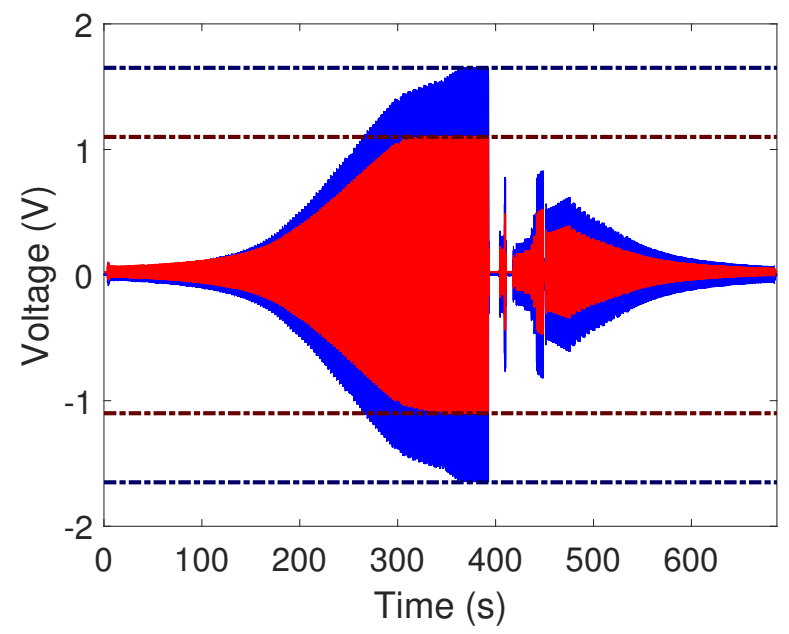

Figure 17: Signals recorded in the MCU for a nonlinear PTVA at $f=1.2 \mathrm{~N}$ : input voltage $(-)$ and its saturation limits at $\pm 1.65 \mathrm{~V}$ (---), output voltage $(-)$ and its saturation limits at $\pm 1.1 \mathrm{~V}(-\cdot-)$.

nonlinear capacitance was computed based on this assumed restoring force using the formulas from [22].

Figure 19 displays the corresponding NFRs. In both cases, a clear detuning is observed compared to Figure 15, which confirms the fact that a cubic capacitance is the optimal choice for a cubic nonlinearity in the host structure, which, in turn, validates the adopted principle of similarity. The detuning can be understood from Figure 18, where the stiffness is first overestimated (resp. underestimated) and then underestimated (resp. overestimated) for a quadratic (resp. quintic) nonlinearity, leading to the same trend for the absorber frequency. 


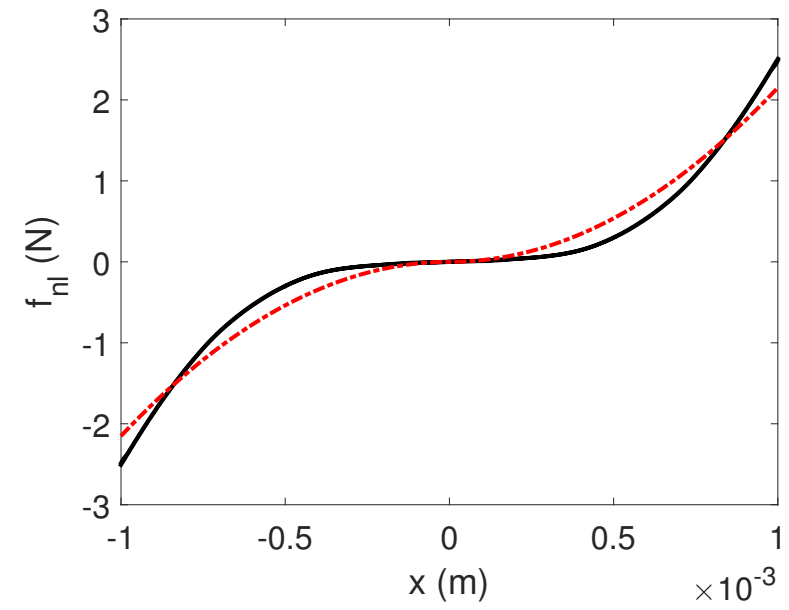

(a)

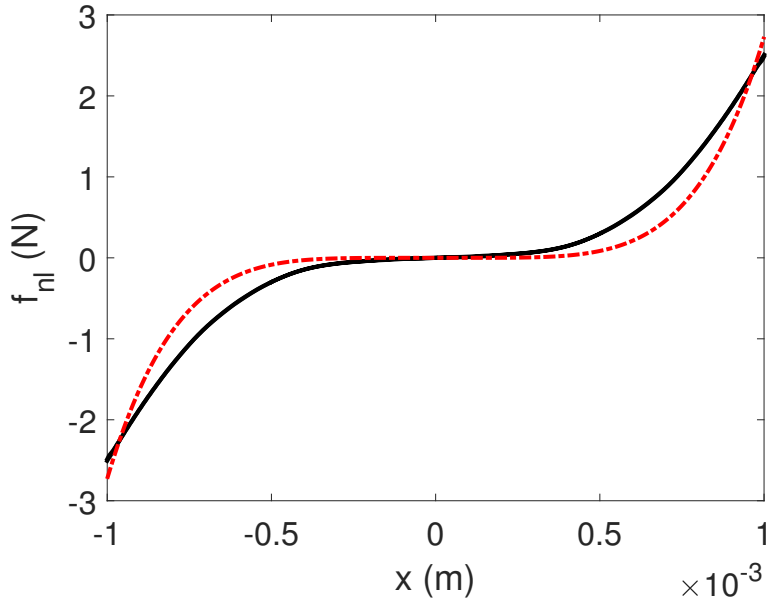

(b)

Figure 18: Fitting the cubic nonlinearity of the thin lamina with a quadratic (a) or a quintic (b) nonlinearity: measurements $(\bullet)$ and fitted model (---).

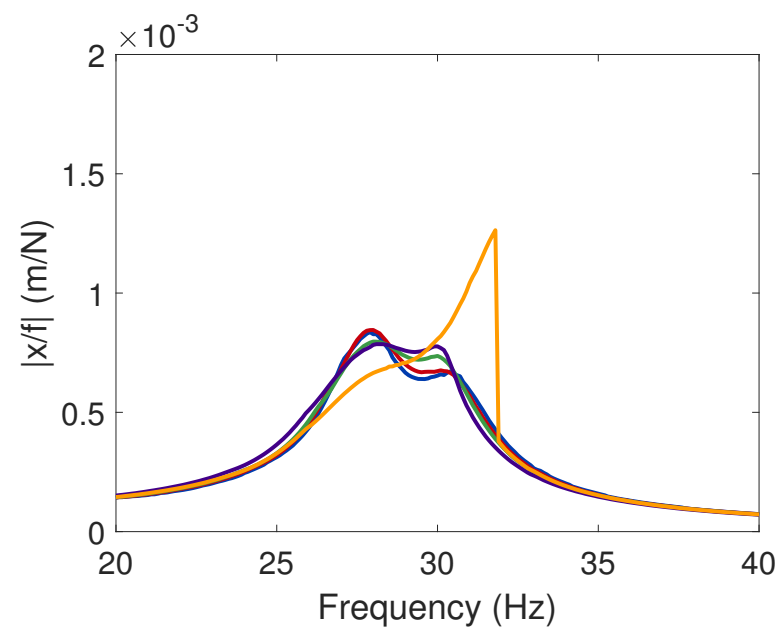

(a)

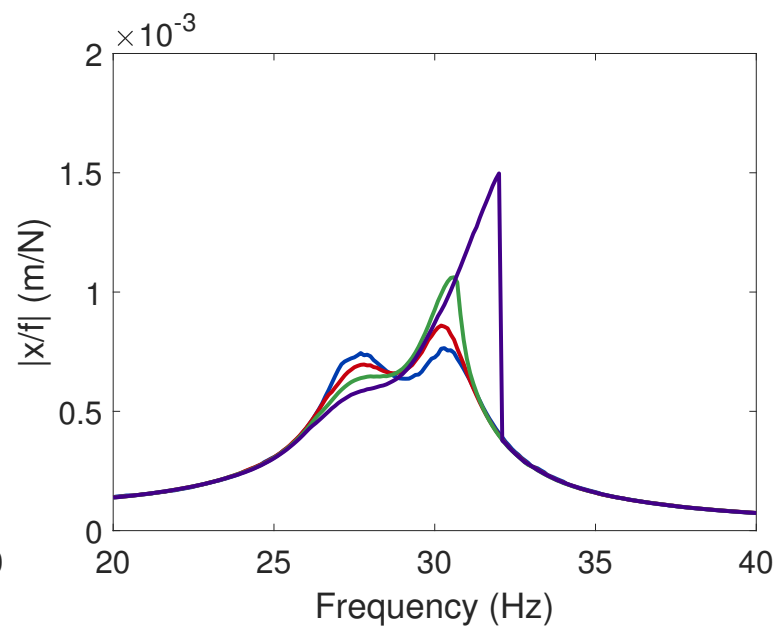

(b)

Figure 19: Experimental NFRs of the nonlinear beam with a digital PTVA with quadratic (a) or quintic (b) capacitance: $0.2 \mathrm{~N}(-), 0.4 \mathrm{~N}(-), 0.6 \mathrm{~N}(-), 0.8 \mathrm{~N}(-$ ), and $1.0 \mathrm{~N}(-)$.

\subsection{Parametric study}

Given the ease with which the parameters of a digital absorber can be modified, performing a parametric study was relatively straightforward. A first parametric study was carried out at a low-level excitation $(0.2 \mathrm{~N})$. In Figure 20, the great sensitivity with respect to the inductance is visible, as a $5 \%$ change leads to a $30 \%$ relative difference in maximum amplitude. Figure 21 shows that the sensitivity on the resistance is much less pronounced. As announced in Subsection 5.2, decreasing the resistance by $10 \%$ has almost no effect on the maximum amplitude. 


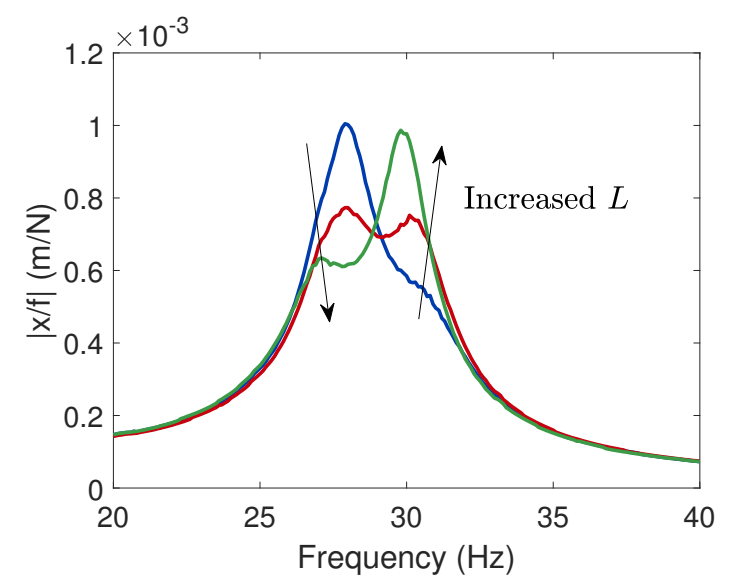

Figure 20: Experimental NFRs of the nonlinear beam with a nonlinear PTVA at $f=0.2 \mathrm{~N}$ when the nominal inductance is multiplied by $0.95(-), 1.0(-)$ and $1.05(-)$.

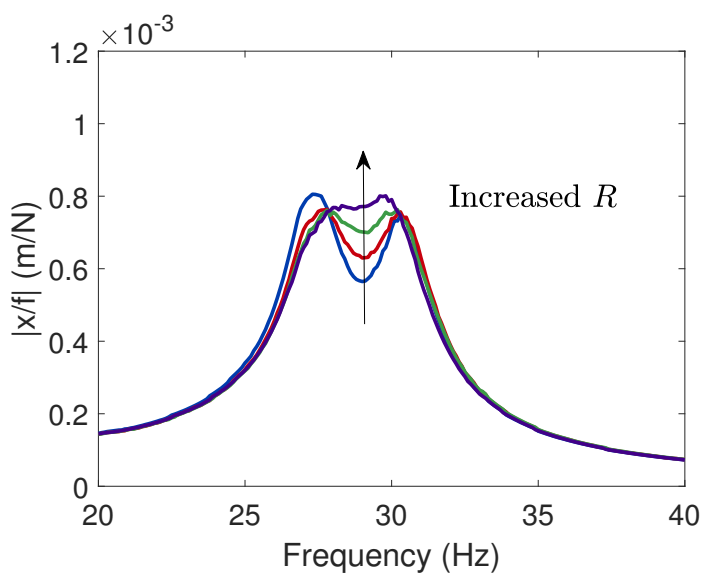

Figure 21: Experimental NFRs of the nonlinear beam with a nonlinear PTVA at $f=0.2 \mathrm{~N}$ when the nominal resistance is multiplied by $0.8(-), 0.9(-), 1.0(-)$ and $1.1(-)$.

A second parametric study was performed at $0.6 \mathrm{~N}$. Figure 22 shows that the nominal nonlinear capacitance (i.e., without the correction for the nonlinearity of the piezoelectric transducer) cannot enforce equal peaks. It is only when the nominal value is multiplied by 2 that equal peaks are obtained. A too large nonlinear capacitance is also detrimental to performance. When comparing Figure 20 and Figure 22, we see that the relative changes in inductance value give rise to much higher detuning than the same relative change in the value of the capacitance.

Finally, Figure 23 shows what happens when the resistance is varied in a nonlinear regime of motion. Qualitatively, the conclusions are the same as in Figure 21, although quantitatively, an increased resistance appears to hinder more the damping ability of the shunt circuit in nonlinear regimes of motion. 


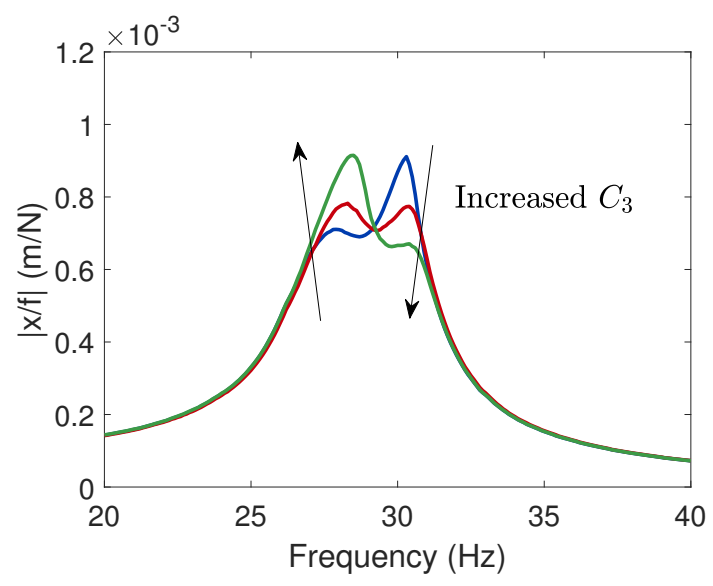

Figure 22: Experimental NFRs of the nonlinear beam with a nonlinear PTVA at $f=0.6 \mathrm{~N}$ when the nominal nonlinear capacitance is multiplied by $1.0(-), 2.0(-)$ and $3.0(-)$.

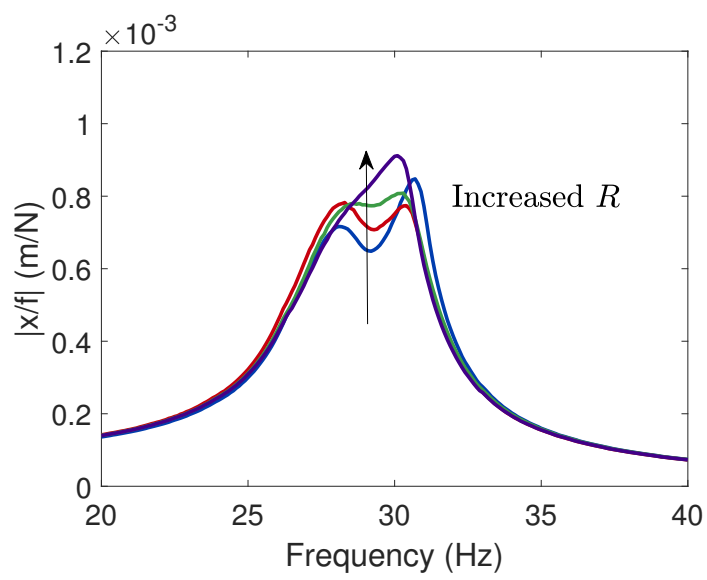

Figure 23: Experimental NFRs of the nonlinear beam with a nonlinear PTVA at $f=0.6 \mathrm{~N}$ when the nominal resistance is multiplied by $0.8(-), 0.9(-), 1.0(-)$ and $1.1(-)$.

\section{Conclusion}

This study presented a digital piezoelectric tuned vibration absorber. Due to its inherent flexibility, this absorber can synthesize linear and nonlinear shunt circuits (with arbitrary mathematical forms for the nonlinearity). The experimental demonstration on a structure with hardening nonlinear behavior showed the superiority of the digital nonlinear absorber over its linear counterpart. It also served to validate the adopted principle of similarity.

This work constitutes a step toward the implementation of absorbers able to effectively mitigate the vibrations of real-life, nonlinear structures. In this framework, digital absorbers constitute an attractive solution, as they are easy to program and to fine-tune. Future works may involve the generalization of this absorber to make it able to control multiple nonlinear structural resonances. 


\section{Acknowledgments}

The authors would like to acknowledge the financial support of the SPW (WALInnov grant 1610122).

\section{References}

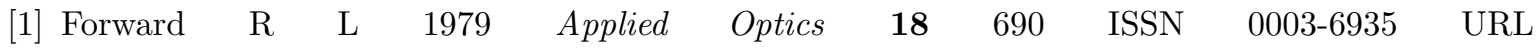
https://www . osapublishing. org/abstract. cfm?URI=ao-18-5-690

[2] Hagood N and von Flotow A 1991 Journal of Sound and Vibration 146 243-268 ISSN 0022460X URL https://linkinghub.elsevier.com/retrieve/pii/0022460X91907629

[3] Soltani P, Kerschen G, Tondreau G and Deraemaeker A 2014 Smart Materials and Structures $\mathbf{2 3} 125014$ ISSN 0964-1726 URL http://stacks. iop.org/0964-1726/23/i=12/a=125014?key=crossref . 798743ce34b627bed945b046d8a6b51a

[4] Ikegame $\mathrm{T}$, Takagi $\mathrm{K}$ and Inoue $\mathrm{T} 2019$ Journal of Vibration and Acoustics $141 \quad 031015$ ISSN 1048-9002 URL http://vibrationacoustics . asmedigitalcollection. asme.org/article. aspx?doi=10.1115/1.4042819

[5] Gripp J and Rade D 2018 Mechanical Systems and Signal Processing 112359 383 ISSN 08883270 URL https://doi.org/10.1016/j.ymssp.2018.04.041 https://linkinghub.elsevier.com/retrieve/pii/S0888327018302437

[6] Den Hartog J P 1985 Mechanical vibrations (Courier Corporation) ISBN 0070163898

[7] Fahy F and Schofield C 1980 Journal of Sound and Vibration 72 365-378 ISSN 0022460X URL https://linkinghub.elsevier.com/retrieve/pii/0022460X80903831

[8] Khavasi A 2015 Journal of the Optical Society of America B 321941 ISSN 0740-3224 URL https://www.osapublishing.org/abstract.cfm?URI=josab-32-9-1941

[9] Arsanjani A, Biabanifard M and Abrishamian M S 2019 Superlattices and Microstructures 128 157-169 ISSN 07496036 URL https://doi.org/10.1016/j.spmi.2019.01.020 https://linkinghub.elsevier.com/retrieve/pii/S074960361831989X

[10] Biabanifard M and Abrishamian M S 2018 Applied Physics A: Materials Science and Processing 124826 ISSN 14320630 URL http://link.springer.com/10.1007/s00339-018-2248-3

[11] Hui Y, Gomez-Diaz J S, Qian Z, Alù A and Rinaldi M 2016 Nature Communications 711249 ISSN 2041-1723 URL http://www.nature.com/articles/ncomms11249

[12] Agnes G S and Inman D J 1996 Smart Materials and Structures 5 704-714 ISSN 0964-1726 URL http://stacks.iop.org/0964-1726/5/i=5/a=018?key=crossref . 1ae9e6cd0aa97b0ac65119847b1ad283

[13] Richard C, Guyomar D, Audigier D and Bassaler H 2000 Enhanced semipassive damping using continuous switching of a piezoelectric device on an inductor vol 3989 ed Hyde $\mathrm{T} \quad \mathrm{T}$ pp $288-299$ ISSN $0277786 \mathrm{X}$ URL http://proceedings. spiedigitallibrary.org/proceeding. aspx?articleid=924877

[14] Zhou B, Thouverez F and Lenoir D 2014 Journal of Sound and Vibration 333 2520-2542 ISSN 0022460X URL http://dx.doi.org/10.1016/j.jsv.2013.12.019 https://linkinghub.elsevier.com/retrieve/pii/S0022460X13010596

[15] Soltani P and Kerschen G 2015 Smart Materials and Structures $\mathbf{2 4} 075015$ ISSN 0964-1726 URL http://dx.doi.org/10.1088/0964-1726/24/7/075015 http://stacks.iop.org/0964-1726/24/i=7/a=075015?key=crossref . 2f 9ebcbf60c9b51319ea2453102f $1 \mathrm{ff} 4$

[16] Pai P, Wen B, Naser A and Schulz M 1998 Journal of Sound and Vibration 215 273-296 ISSN 0022460X URL https://linkinghub.elsevier.com/retrieve/pii/S0022460X98916121

[17] Oueini S S and Nayfeh A H 2000 Journal of Vibration and Control 6 999-1016 ISSN 1077-5463 URL http://journals.sagepub.com/doi/10.1177/107754630000600703

[18] Lossouarn B, Deü J F and Kerschen G 2018 Philosophical Transactions of the Royal Society 
A: Mathematical, Physical and Engineering Sciences 37620170142 ISSN 1364-503X URL http://rsta.royalsocietypublishing.org/lookup/doi/10.1098/rsta.2017.0142

[19] Silva T M, Clementino M A, De Marqui C and Erturk A 2018 Journal of Sound and Vibration 437 68-78 ISSN 0022460X URL https://doi.org/10.1016/j.jsv.2018.08.038 https://linkinghub.elsevier.com/retrieve/pii/S0022460X18305455

[20] Lo Feudo S, Touzé C, Boisson J and Cumunel G 2019 Journal of Sound and Vibration $438 \quad 33-53$ ISSN 0022460X URL https://linkinghub.elsevier.com/retrieve/pii/S0022460X18305868

[21] Fleming A, Behrens S and Moheimani S 2000 Electronics Letters 361525 ISSN 00135194 URL https://digital-library.theiet.org/content/journals/10.1049/el_20001083

[22] Habib G and Kerschen G 2016 Physica D: Nonlinear Phenomena 332 1-8 ISSN 01672789 (Preprint 1606.01244) URL http://dx.doi.org/10.1016/j.physd.2016.06.001 https://linkinghub.elsevier.com/retrieve/pii/S0167278915300580

[23] Detroux T, Renson L, Masset L and Kerschen G 2015 Computer Methods in Applied Mechanics and Engineering 296 18-38 ISSN 00457825 (Preprint 1604.05621) URL http://dx.doi.org/10.1016/j.cma.2015.07.017 https://linkinghub.elsevier.com/retrieve/pii/S0045782515002297

[24] Lossouarn B, Aucejo M, Deü J F and Multon B 2017 Sensors and Actuators A: Physical 259 68-76 ISSN 09244247 URL http://dx.doi.org/10.1016/j.sna.2017.03.030 https://linkinghub.elsevier.com/retrieve/pii/S0924424716309785

[25] Park C H and Inman D J 2003 Shock and Vibration 10 127-133 ISSN 1070-9622 URL http://www.hindawi.com/journals/sv/2003/863252/abs/

[26] Matten G, Collet M, Cogan S and Sadoulet-Reboul E 2014 Procedia Technology 15 84-89 ISSN 22120173 URL http://linkinghub.elsevier.com/retrieve/pii/S2212017314001522 https://linkinghub.elsevier.com/retrieve/pii/S2212017314001522

[27] Horowitz P and Hill W 2015 The Art of Electronics 3rd ed (Cambridge University Press) ISBN 978-0-521-37095-0

[28] Apex Microtechnology, Inc 2012 PA78 Power Operational Amplifier URL https://www . apexanalog.com/resources/products/pa78u.pdf

[29] Adams D and Allemang R 2000 Mechanical Systems and Signal Processing 14 637-656 ISSN 08883270 URL https://linkinghub.elsevier.com/retrieve/pii/S0888327000912925 